\section{API para control de asistencia con reconocimiento facial usando OpenCv.JS}

\author{
API for attendance control with facial recognition using \\ OpenCv.JS
}

\section{Ingrid Angélica García Torres* \\ Ximena Trujillo Borja* \\ Javier Domínguez De La Torre* \\ William Navas Espín*}

\section{RESUMEN}

Mediante investigación la, el control de asistencia de aquellos estudiantes de las instituciones educativas se toma de forma periódica y a través de procesos manuales, para superar esta situación se realizó la presente investigación que incluye la revisión de tecnologías de reconocimiento facial para diseñar una API que se encargue del registro y control de asistencia de los estudiantes, que pueda ser utilizado en múltiples sistemas, se utilizó una metodología basada en el método bibliográfico y cuantitativamente, empleando una encuesta a docentes de la Universidad Estatal del Sur de Manabí evaluando el interés de esa población en el diseño propuesto, se ejecutó la revisión de tecnologías previamente utilizadas para diseño de API, se investigó la evolución de software empleados en servicios a fines, se empleó el uso librerías gratuitas como OpenCv y Nodejs. Lográndose con ella la construcción del diseño para el uso del control de asistencia acoplable a sistemas actuales y con tecnología moderna.

Palabras clave: Opencv, API, Reconocimiento facial, Estudiantes

\footnotetext{
* Master, Universidad de Guayaquil, Guayaquil, Ecuador, ingrid.garciat@ug.edu.ec, https://orcid.org/0000-0003-0333-7525

* Master,Universidad de Guayaquil, Guayaquil, Ecuador, ximena.trujillob@ug.edu.ec, Guayaquil, Ecuador https://orcid.org/0000-0003-I520-9096

* Master,Universidad de Guayaquil, Guayaquil, Ecuador, luis.dominguezt@ug.edu.ec, Guayquil, Ecuador https://orcid.org/0000-0002-4287-907।

* Master, Universidad de Guayaquil, Guayaquil, Ecuador, william.navase@ug.edu.ec, https://orcid.org/0000-0002-8492-9997
}

\section{REVISTATECNOLÓGICA ciencia y educación Edwards Deming}

ISSN: 2600-5867

\section{Atribución/Reconocimiento-NoCo} mercial- Compartirlgual 4.0 Licencia Pública Internacional - CC

\section{BY-NC-SA 4.0}

https://creativecommons.org/licenses /by-nc- sa/4.0/legalcode.es

Editado por: Tecnológico Superior Corporativo Edwards Deming Enero - Julio Vol. 5 - I - 202। https://revista-edwardsdeming.com/index.php/es e-ISSN: 2576-097।

Recibido: 19 Marzo 2020

Aprobado: 24 Diciembre, 2020

Pag 29-39 


\section{ABSTRACT}

Through research, the attendance control of those students of educational institutions is taken periodically and through manual processes, to overcome this situation the present investigation was carried out that includes the review of facial recognition technologies to design an API that is In charge of the registration and control of student attendance, which can be used in multiple systems, a methodology based on the bibliographic method and quantitatively was used, using a survey of teachers from the State University of the South of Manabí evaluating the interest of that population In the proposed design, the review of technologies previously used for API design was carried out, the evolution of software used in services was investigated, the use of free libraries such as OpenCv and Nodejs was used. Achieving with it the construction of the design for the use of assistance control that can be coupled to current systems and with modern technology.

Keywords: Opencv, API, Face recognition, Students.

\section{INTRODUCCIÓN}

Computer Vision, que generalmente se abrevia como CV, se caracteriza por ser un campo muy amplio de la inteligencia artificial que busca crear métodos para ayudar a las computadoras a "ver" y obtener la sustancia de imágenes avanzadas como fotos y videos. El objetivo de Computer Vision es extraer datos valiosos de las imágenes.

Esto ha demostrado lo sorprendentemente desafiante que es; ha poseído miles de mentes brillantes e inventivas durante las últimas cuatro décadas, y a pesar de esto todavía se está lejos de poder construir una "máquina de ver" de propósito general.

El reconocimiento facial es un método para identificar o verificar la identidad de un individuo mediante la detección de patrones faciales. Los sistemas de reconocimiento facial se pueden utilizar para identificar personas en fotos, videos o en tiempo real. La policía también puede usar dispositivos móviles para identificar personas durante las paradas policiales.

Pero los datos de reconocimiento facial pueden ser propensos a errores, lo que puede implicar a las personas por delitos que no han cometido. El software de reconocimiento facial presenta falencias para reconocer a los afroamericanos y otras minorías étnicas, mujeres y jóvenes, a menudo identificándolos erróneamente o no identificándolos, impactando de manera dispar a ciertos grupos (E3FF, 20I4).

El reconocimiento facial abarca muchos ámbitos de tecnología incluso con los controles de acceso como los biométricos, y son adaptables a cualquier sistema pudiendo ser web en cualquier sistema operativo,

Los Biométricos se utiliza para la seguridad y / o autenticación de transacciones. Un sistema biométrico es una forma efectiva de verificar la identidad de una persona. Se utilizan en aeropuertos, investigaciones forenses, edificios seguros y gestión de la fuerza laboral. Tomando que las unidades administrativas han obtenido beneficio de este tipo de tecnología. En este trabajo se propone el diseño de un api usando inteligencia artificial mediante aprendizaje por tareas como Deep Learning y derivados. Debido a esto se usa 
un sistema biométrico sencillo y se diseñará un sistema de reconocimiento capaz de aprender por sí mismo, con una auto eficiencia y a bajo coste.

Este diseño utilizará lenguajes de programación de alto nivel, con lado al cliente usando datos y requerimientos de manera asíncrona como lo es nodejs y usando una librería de computer visión como lo es OPENCV.JS que ayudara con la forma y reconocimiento del rostro de una manera eficiente. El presente trabajo consta de 3 etapas:

Etapa I: El diseño del backend como etapa I.

Etapa 2: Uso de Nodejs y una base de datos robusta como lo es MySQL para un mejor control y flujo de datos, como intermedio entre el backend y frontend.

Etapa 3: Uso de un framework express basado en Nodejs para un diseño de interfaces más intuitiva a nivel de usuario.

\section{MATERIALES Y MÉTODOS}

\section{Modalidad de la Investigación}

Con la finalidad de tramitar la problemática planteada anteriormente se propone el diseño del API para detectar los rostros de los estudiantes de la facultad de Tecnología de Información de la cuidad de Jipijapa esta herramienta mostrará la eficiencia en sistemas de reconocimiento facial al detectar rostros y tomar la asistencia en el cual se hizo una herramienta en beneficio a los profesores cuyo sistema facilita el proceso normal en la toma de asistencias.

\section{Enfoque de la investigación}

Para el diseño del API, se considera los siguientes aspectos importantes que son:

- Investigación Técnica

- Investigación Cuantitativa

- Investigación Cualitativa

Debido al estudio de esta investigación de los diferentes sistemas de control de asistencia, existen distintos tipos de reconocimiento facial que ayudan a sistemas de control de accesos y actualmente no existen sistemas que implementen en comunicación con sistemas actuales educativos.

\section{Investigación Técnica}

En base a las pruebas de diseño se realizó una simulación durante la investigación en la que se determinó lo viable del uso de herramientas open source (código abierto), estas herramientas tecnológicas brindan una mejor viabilidad al momento de desarrollar dicho API con las funcionalidades que requieren para el control de asistencia de estudiantes. Los recursos para la funcionalidad tanto como en hardware o software se encuentran en la mayor parte del mercado actual.

El uso de estas herramientas comprende de dos etapas una de ellas el backend que básicamente compone el cuerpo del API, y el frontend como lo es NODEJS. Uno de los componentes del backend es MySQL en la cual se almacena los datos de los estudiantes 
para su detección. MySQL es un gestor que viene incluido con XAMP, mediante esto facilita el manejo a nivel de servidor y gestión de datos de la base de datos del API. En el frontend usa NODEJS que es un intermedio de comunicación entre la parte del backend y el frontend, que usara los datos que guarda y los mostrara en una página basada en HTML y JavaScript, y por último para la parte principal del articulo para el reconocimiento de los rostros en donde se usa OpenCv con JavaScript.

Tabla I Recurso de Software

\section{Herramientas}

\section{Característica}

SO (Sistema Operativo)

Base de Datos

Servidor de Aplicaciones

Framework

Lenguaje de Programación
Multisistema portable

MySQL

Apache/ Nodejs

Bootstrap, JQuery

TypeScript/ JavaScript

\section{Información tomada de la investigación directa. Elaborado por Investigadores}

Una de las ventajas de usar este tipo de tecnologías de código abierto es lo escalable que puede ser usar Nodejs y los beneficios de su escalabilidad a nivel estructural facilitan como anteriormente en la tabla 2 sobre el recurso del software. El gestor de base de datos amigable adaptable a múltiples IDE para el manejo de información. Servidor web de Nodejs que da como ventaja ser asíncrono en comunicación con el backend y el frontend. La experiencia UIX a nivel de usuario que brinda Bootstrap. En la tabla 3 se detalla uno de los beneficios a nivel de hardware que brinda Nodejs como servidor.

Tabla 2 Recurso Hardware - Servidor

Características

$$
\begin{gathered}
\text { Procesador } \\
\text { Memoria }
\end{gathered}
$$

Disco Duro

Monitor "I5"

\section{Especificaciones}

\section{Core i3 6ma Generación \\ 4 Gb RAM \\ I TB}

Portátil 
Información tomada de la investigación directa. Elaborado por Investigadores

\section{Investigación Cuantitativa}

El uso de esta información se obtiene la investigación directa, que con el uso de esta metodología permitirá recopilar, analizar la información por medio de datos de un estudio previamente hecho, las herramientas tecnológicas que se usan en este diseño ayudaran a obtener resultado sobre el funcionamiento de la simulación del API.

\section{Investigación Cualitativa}

El docente usara la aplicación que disponen el conocimiento necesario para el funcionamiento del sistema, se realizara una encuesta a los docentes con dichas preguntas elaboradas de manera objetiva con la finalidad de enfocarse en el diseño del API.

\section{Población que colaboró para ser efectiva la investigación}

La población es el conjunto de personas que se encuentran en un sitio determinado, me permitirá realizar generalizaciones debido al tamaño de Docentes de la Carrera de Tecnología de Información de la Universidad Estatal del Sur de Manabí de la ciudad de Jipijapa mediante el uso del muestreo no probabilístico, se calculó de la siguiente manera:

\section{Dónde:}

$$
n=\frac{N}{1+\frac{e^{2}(N-1)}{z^{2} p q}}
$$

$\mathbf{n}=$ tamaño de la muestra

$\mathbf{N}=$ tamaño conocido de la población

$\mathbf{z}=$ nivel de confianza $92 \%$

e= error máximo permitido $6 \%$

$\mathbf{p}=$ Probabilidad a favor

$\mathbf{q}=$ Probabilidad en contra

$$
\begin{aligned}
& \mathbf{N}=180 \quad z=1.96 \quad e=6 \quad p=0.50 \quad q=0.50 \quad n=? \quad \text { Datos: } \\
& n=\frac{180}{1+\frac{0.05^{2}(180-1)}{1.96^{2}(0.50)(0.50)}} \\
& n=\frac{180}{1+\frac{0.0025(179)}{3.8416(0.25)}}
\end{aligned}
$$




$$
n=98
$$

$$
\begin{gathered}
n=\frac{180}{1+\frac{0.0475}{0.9604}} \\
n=\frac{180}{1.0494}
\end{gathered}
$$

Por lo tanto, será necesario encuestar no menos de 98 usuarios para tener una fiabilidad en la información del $92 \%$.

Durante el procesamiento de la información la cantidad de información que se obtuvo en la encuesta propuesta, en todas las descripciones se hicieron con la herramienta de formulario de Google que da beneficios como estadísticas de la encuesta en porcentaje de respuestas, representaciones en tablas y graficas que se necesitan para el análisis de interrogantes.

\section{Ejecución de encuestas}

Aplicando el método de investigación cuantitativa, se procedió a utilizar el instrumento encuesta, mediante la cual la misma que fue enviada a los docentes explicando previamente los conceptos de API y el objetivo del diseño.

El desarrollo de la encuesta como se mencionó con anterioridad se estableció como población a docentes de una institución educativa en la cual se realizó el proceso de recolección de información.

\section{Análisis de las encuestas}

Este modelo va dirigido a docentes en general que usan el control de asistencia de manera manual o usando apartados de sistemas educativos en general. Este modelo permitirá conocer una posible demanda en beneficio a la prueba y simulación de este diseño dentro de la ciudad de Jipijapa.

Se realizo el proceso de encuesta a 20 Docentes que mostraron su punto de vista de acuerdo al beneficio del diseño de este articulo con finalidad de obtener resultados para llegar a las conclusiones debidas. Con el fin a este proceso se realizó 8 preguntas en consecuencia, a esto las preguntas son las siguientes:

\section{¿Qué método usa usted frecuentemente para la toma de asistencia a estudiantes?}

Tabla 3 Métodos de toma de asistencia

\begin{tabular}{ccc}
\hline Opciones de Respuestas & Docentes & Porcentajes \\
\hline $\begin{array}{c}\text { Excel } \\
\text { Sistemas Integrados en } \\
\text { plataforma educativa }\end{array}$ & 63 & $63 \%$ \\
\end{tabular}


Bloc de Notas

Otros

Total

Información tomada de la investigación directa, Elaborado por Investigadores.

100 respuestas

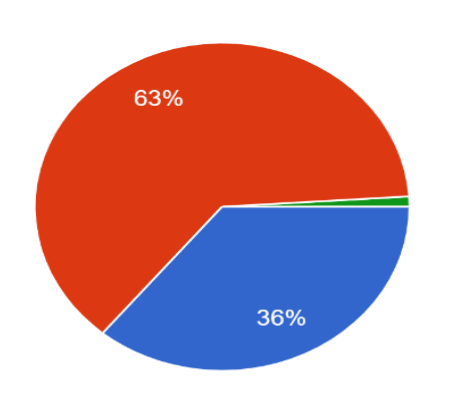

$0 \quad 0 \%$

I

$1 \%$

$100 \%$
Excel
Sistemas Integrados en plataforma educativa
Bloc de Notas
Otros

Figura I Métodos de toma de asistencia a estudiantes, Información tomada de la investigación directa. Elaborado por Investigadores

Según los datos que se obtuvieron en la figura, se dio como resultado que los docentes usan en su mayoría (63\%) como método de toma de asistencia la plataforma educativa o Excel, solo el $36 \%$ emplea otros métodos de tipo manual o semi manual.

\section{Esquema general de simulación}

Para el desarrollo de una simulación en base al diseño de este API, es necesario ver que se compone de dos partes la parte del backend y la parte del frontend.

El backend se compone de múltiples servicios como se ve en la figura 2 .

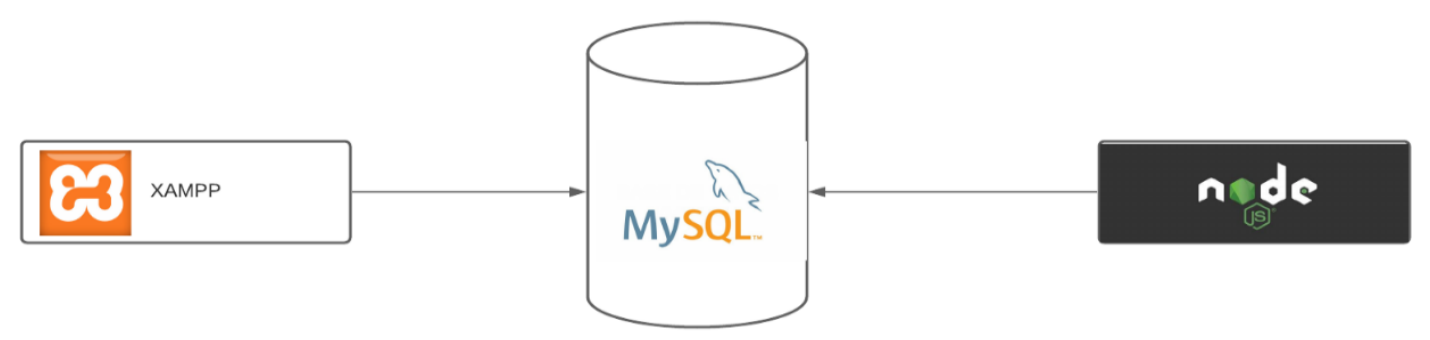

Figura 2 Estructura Backend Información tomada de la investigación directa. Elaborado por Investigadores. 
El frontend que se compone de la parte visual del API como se observa ver en la figura 3.

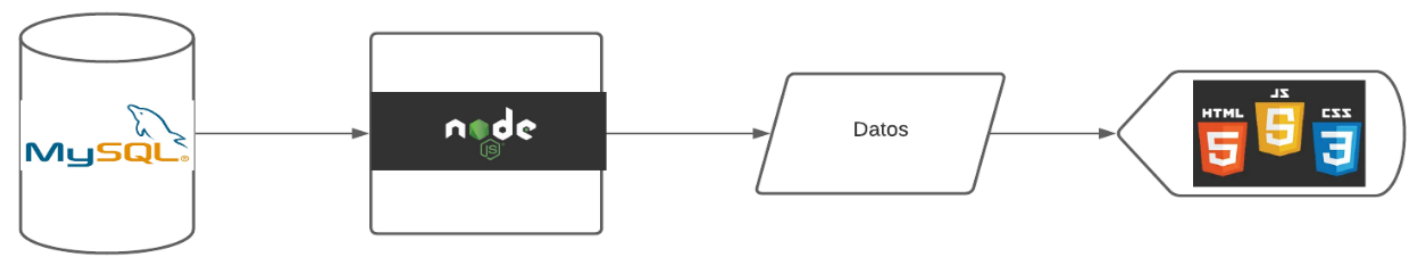

Figura 3. Estructura Frontend. Información tomada de la investigación directa.

Elaborado por Investigadores

\section{RESULTADOS}

\section{Descripción de funcionalidades}

Al evaluar el todo sentido de la funcionalidad es necesario explicar explícitamente cada botón y clases que se encuentran en el Sidebar, el que cual se menciona anteriormente en el que se compone de numerosas funcionalidades.

\section{Reconocimiento facial único por imagen}

Una de las variantes en el sistema es el reconocimiento facial mediante una imagen guardada previamente en la computadora, para un mejor funcionamiento de este.

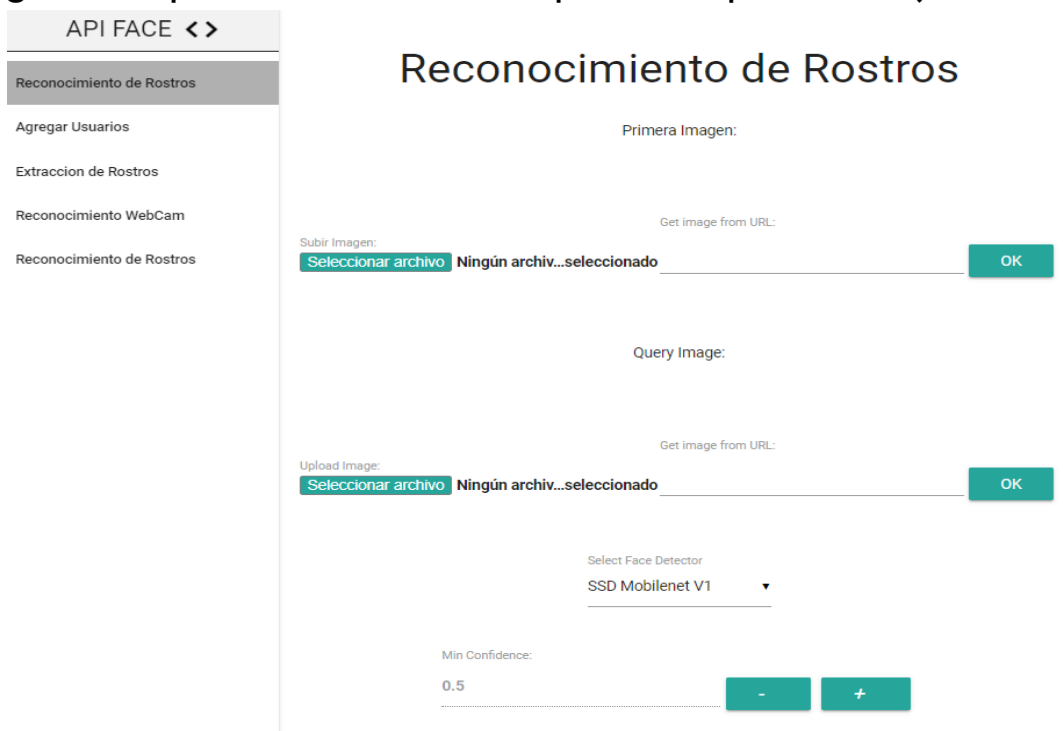

Figura 4 Sección de reconocimiento de rostros por imagen. Información tomada directa de la investigación. Elaborado por Investigadores. 
Como se puede observar en la figura la vista tiene como vía principal reconocer el rostro mediante varios detectores SSD Mobilenet VI y TinyFaceDetector, se puede usar mediante el url de una imagen o subir compatibles de imágenes como jpg, png, etc.

El reconocimiento se da de manera asíncrona con el sistema sin necesidad de recargar el navegador y muestra a la imagen subida el reconocimiento del rostro como se puede observar en la figura 5.

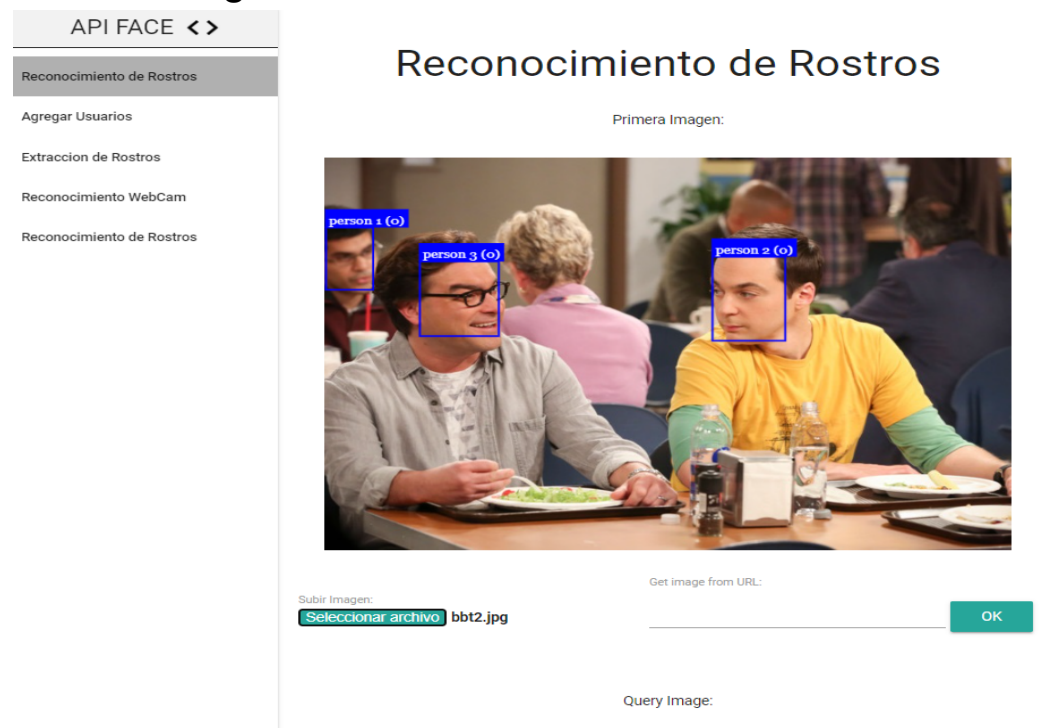

Figura 5 Rostros reconocidos por funcionalidad de imagen. Información tomada directa de la investigación. Elaborado por Investigadores.

\section{DISCUSIÓN}

El formulario hecho para el reconocimiento previo del rostro a través de esto la funcionalidad de los demás módulos no podrían funcionar sin un registro de cada persona. Los campos que contienen son: nombre, apellidos, y un input de imágenes en donde subes el rostro para reconocer, a través de un botón se realiza el guardado de la imagen y el resto de los datos mencionados anteriormente.

Este módulo es el principal para el reconocimiento y toma de asistencias a estudiantes, su funcionalidad es por demás la más importante en el desarrollo del API, su uso es exactamente como observa anteriormente, la subida de la imagen puede ser por imagen url.

En el módulo de extracción de rostros, se reconocen los rostros que previamente estén guardados en el sistema. El uso del botón de personas reconocidas muestra la 
salida de comunicación con sistemas, el formato JSON de las personas reconocidas en la extracción que cumplan con las características que definen a las personas previamente, además de la posibilidad de usar los dos detectores TinyFace Detector y MobilNet para un mejor funcionamiento en el reconocimiento.

\section{REFERENCIAS}

Tarazona Ospina, I., \& MSc. Daniel, V. (20I5). RECONOCIMIENTO FACIAL BASADO EN EIGENFACES, LBHP Y FISHERFACES EN LA BEAGLEBOARD-xM. Revista colombiana de tecnologias de avanzada, I48, 149.

Alvarez, M. A. (I9 de Noviembre de 2015). Desarrolloweb.com. Obtenido de ¿Que es python?: https://desarrolloweb.com/articulos/I325.php

BBVAOPEN4U. (23 de Marzo de 2016). BBVAOPEN4U. Obtenido de https://bbvaopen4u.com/es/actualidad/api-rest-que-es-y-cuales-son-sus-ventajasen-el-desarrollo-de-proyectos

COIP. (2014). Libro de los Artículos del Código Orgánico Penal. Obtenido de https://tbinternet.ohchr.org/Treaties/CEDAW/Shared\%20Documents/ECU/INT _CEDAW_ARL_ECU_18950_S.pdf

E3FF. (2 de Enero de 20I4). E3FF. Obtenido de https://www.eff.org/es/pages/facerecognition

Emscripten. (20I5). Emscripten. Obtenido de https://emscripten.org/

Enatec. (24 de Mayo de 20l0). Enatec. Obtenido de https://einatec.com/control-accesobiometricol

Gamboa, M., Barros, L., \& Barros, C. (2019). Childhood Aggressiveness, Learning and Self-Regulation in Primary Students. Luz. Revista Electrónica Trimestral de La Universidad de Holguín, 53(9), 1689-1699. https://luz.uho.edu.cu/index.php/luz/article/view/743/637

Gebhart, A. (27 de Marzo de 2019). Cnet. Obtenido de Cnet: https:/www.cnet.com/es/noticias/reconocimiento-facial-apple-amazon-googleai/

Gendra, M. (4 de Mayo de 2010). https://www.marianogendra.com.ar/Articulos/que-es-unaapi. Obtenido de https://www.marianogendra.com.ar/Articulos/que-es-una-api 
Martinez, N. (4 de Junio de 2019). IEBS. Obtenido de https://www.iebschool.com/blog/reconocimiento-facialtecnologia/\#\%C2\%BFQue_es_el_reconocimiento_facial

Maza, E. A., \& Goyes Mosquera, J. J. (20I5). Repositorio Digital. Obtenido de Sistema de información y control de asistencia del personal para Facultad de Ingeniería.: http://www.dspace.uce.edu.ec/handle/25000/496 